УДК 621.396 .67

\title{
ВЛИЯНИЕ МЕДИ И ГРАФЕНА НА БИКОНИЧЕСКУЮ АНТЕННУ В ДИАПАЗОНЕ ЧАСТОТ 1,2 ГГЦ
}

\author{
Р. ПРАСАННА, Н. М. МАСУДХУ БАНУ
}

Научно-исследовательский институт науки и технологий Вел Тек им. др. Сагунтала Рангараджан, Ченнаи, Индия

\begin{abstract}
Аннотация. В статье сравнивается влияние меди и графеновых проводящих чернил на характеристики плоской биконической (bow-tie) антенны. Полосковые структуры антенны моделируются с использованием программного пакета HFSS. Спроектированная антенна резонирует на частоте около 1,2 ГГц с коэффициентом отражения ниже -10 дБ. Предлагаемая модифицированная конструкция bow-tie aнтенны имеет ширину рабочей полосы 15 (расчет), 12 (медь, эксперимент) и 13,5 МГц (графен, эксперимент), соответственно, что позволяет ее использовать в LTE (QUALCOMM), WiMAX, Wi-Fi диапазонах. Эта модифицированная конструкция состоит из различных типов щелей, введенных в разных местах биконической антенны, чтобы получить желаемую ширину полосы и улучшить эффективность антенны. Предложенная антенна рассчитана для работы в диапазоне УВЧ (ультравысокой частоты) на частоте 1,2 ГГц. По сравнению с обычной bow-tie антенной предлагаемая модифицированная конструкция имеет лучшие характеристики. В работе выполнено сравнение различных рабочих характеристик, таких как потери на отражение, КСВН и полоса для каждой предложенной реализации. Рассчитанные характеристики подтверждены экспериментально для предложенной модели на резонансной частоте.
\end{abstract}

Ключевые слова: модифицированная конструкция биконической антенны; микрополосковая антенна; УВЧ; диапазон ультравысоких частот; щель; полоса пропускания; bow-tie

\section{ВВЕДЕНИЕ}

Антенна-бабочка (bow-tie) — это двухмерная версия биконической антенны, используемой в диапазоне ультравысоких частот (УВЧ) [1].

Микрополосковая антенна-бабочка широко используется в последние годы для исследовательских и инженерных задач благодаря ее малому весу, тонкому профилю, низкой стоимости, хорошим характеристикам, простоте изготовления, компактности и простой геометрии. Bow-tie антенна представляет собой комбинацию двух треугольных участков с микрополосковой подачей питания, изготовленных на подходящей подложке [2]. Хорошие характеристики делают ее пригодной для различных применений, где необходимы малый вес, не- большие размеры, низкая стоимость, высокая эффективность, простота установки и геометрии. Эти особенности полезны в спутниковой связи, телевидении, радиолокации, навигационных системах, радарах, CШП, Wi-Fi, WLAN, WiMAX, беспроводных камерах наблюдения, беспроводных каналах видеосвязи и передатчиках, а также других системах связи [3].

Эти микрополосковые патч-антенны совместимы с различными твердотельными устройствами, такими как генераторы, усилители, переключатели, смесители и другие электронные устройства. Такие модули могут быть добавлены непосредственно на подложку и могут иметь различные резонансные частоты [4]. 


\section{REFERENCES}

1. https://en.wikipedia.org/wiki/Biconical antenna.

2. Bhosale, A. C.; Deshmukh, V. U. "Design of bow-tie microstrip antenna with fractal shape for W-LAN application," IJECT, Vol. 3, No. 4, 2012. URI: http://www.iject.org/vol34/3/amol.pdf.

3. Rahim, M. K. A.; Aziz, M. Z. A. Abdul; Goh, C. S. "Bow-tie microstrip antenna design," Proc. of 13th IEEE Int. Conf. on Networks Jointly held with the 2005 IEEE 7th Malaysia Int. Conf. on Communic., 16-18 Nov. 2005, Kuala Lumpur, Malaysia. IEEE, 2005. DOI: 10.1109/ICON.2005.1635425.

4. Singh, Sukhdeep; Gupta, Vikas; Kumar, Avish. "A review of bow tie antenna and microstrip patch antenna," Int. J. Eng. Res. Tech., Vol. 3, No. 5, 2014. URI: https://www.ijert.org/research/a-review-of-bow-tie-antennaand-microstrip-patch-antenna-IJERTV3IS050786.pdf.

5. Shafiei, M. M.; Moghavvemi, Mahmoud; Mahadi, Wan Nor Liza Wan. "The parametric study and fine-tuning of bow-tie slot antenna with loaded stub," PLoS One, Vol. 12, No. 1, p. e0169033, 2017. DOI: 10.1371 /journal.pone.0169033.

6. Haque, Ahasanul; Reza, Ahmed Wasif; Kumar, Narendra; Ramiah, Harikrishnan. "Slotting effect in designing circular edge bow-tie nano antenna for energy harvesting," Proc. of IEEE Conf. on Open Systems, 24-26 Aug. 2015, Bandar Melaka, Malaysia. IEEE, DOI: 10.1109/icos.2015.7377276. URI:

7. Bandwidth support in LTE standards by QUALCOMM.

https://ransition.fcc.gov/bureaus/oet/tac/tacdocs/meeting71612/ PANEL2.3-GaalQualcomm.pdf.

8 .

http://www.rfwirelessworld.com/calculators/Bowtie-Antenna Calculator.html.

9. Eldek, A. A.; Elsherbeni, A. Z.; Smith, C. E. "Wideband slot bow-tie antennas for radar applications," Proc. of IEEE
Topical Conf. on Wireless Communication Technology, 15-17 Oct. 2003, Honolulu, USA. IEEE, 2003. DOI: 10.1109/WCT.2003.1321597.

10. Chen, Y.-L.; Ruan, C.-L.; Peng, L. "A novel ultra-wideband bow-tie slot antenna in wireless communication systems," PIER Lett., Vol. 1, p. 101-108, 2008. DOI: 10.2528/pierl07112302.

11. Xu, Lina; Li, Li; Zhang, Wenmei. "Study and design of broadband bow-tie slot antenna fed with asymmetric CPW," IEEE Trans. Antennas Propag., Vol. 63, No. 2, p. 760-765, 2015. DOI: $10.1109 /$ tap.2014.2378265.

12. Kundu, Twinkle; Parkash, Davinder. "A triangular patch antenna for UHF band with microstrip feed line for RFID applications," Int. J. Eng. Res. Tech., Vol. 1, No. 8, 2012. URI: https://www.ijert.org/research/a-triangular-patch-antennafor-uhf-band-with-microstrip-feed-linefor-rfid-applications-IJERTV1IS8249.pdf

13. Li, Biao; Yin, Ying-Zeng; Hu, Wei; Ding, Yang; Zhao, Yang. "Wideband dual-polarized patch antenna with low cross polarization and high isolation," IEEE Antennas Wireless Propag. Lett., Vol. 11, p. 427-430, 2012. DOI: 10.1109/lawp.2012.2195149.

14. Tawk, Y.; Kabalan, K. Y.; El-Haji, A.; Christodoulou, C. G.; Costantine, J. "A simple multiband printed bowtie antenna," IEEE Antennas Wireless Propag. Lett., Vol. 7, p. 557-560, 2008. DOI: 10.1109/lawp.2008.2001027.

15. Feng, Guirong; Chen, Lei; Wang, Xinwei; Xue, Xingsi; Shi, Xiaowei. "Broadband circularly polarized crossed bowtie dipole antenna loaded with parasitic elements," IEEE Antennas Wireless Propag. Lett., Vol. 17, No. 1, p. 114-117, 2018. DOI: 10.1109/lawp.2017.2777442.

16. Wu, Ming-Tien; Chuang, Ming-Lin. "Multibroadband slotted bow-tie monopole antenna," IEEE Antennas Wireless Propag. Lett., Vol. 14, p. 887-890. 2015. DOI: 10.1109/lawp.2014.2383441. 